\title{
Reliability and validity of a simple and clinically applicable pain stimulus: sustained mechanical pressure with a spring-clamp
}

\author{
Søren O'Neill ${ }^{1,2,3^{*}}$, Thomas Graven-Nielsen ${ }^{1}$, Claus Manniche ${ }^{2,3}$ and Lars Arendt-Nielsen ${ }^{1}$
}

\begin{abstract}
Background: Generalized hyperalgesia, a widespread increased sensitivity to painful stimuli, has been demonstrated in a range of chronic pain conditions including low-back pain. The evidence suggests, that generalized hyperalgesia may be an important factor in the development of chronicity, but it is not commonly assessed in clinical practice. Whereas a range of tools and procedures for the quantitative sensory testing of pain sensitivity is available for laboratory pain research, most experimental pain stimuli are not well suited for clinical practice. In the current study, a simple and inexpensive mechanical spring-clamp was tested as a potential experimental pain stimulus.

Methods: Ten seconds of mechanical pressure was applied to the thumb of 242 study participants: Healthy volunteers and low-back-pain patients (hospital and primary care). Pain intensity was measured by visual analogue scale before and after conditioned pain modulation by cold-pressor test (CPT). Correlation to pressure pain threshold (PPT) of the infraspinatus muscle and cold-pressor test pain intensity, time to pain onset and time to non-tolerance, was examined. Test/re-test reliability of clamp pain was also assessed and the stimulus-response relationship was examined with a set of 6 different clamps.
\end{abstract}

Conclusions: Clamp pain was sensitive to changes in pain sensitivity provoked by conditioned pain modulation (CPM). Test/re-test reliability of the spring-clamp pain was better for healthy volunteers over a period of days, than for hospital patients over a period of weeks. A strong correlation $(\rho=0.73, P>0.0001)$ was found between clamp force and evoked pain intensity. Correlation to other pain stimuli varied, with a strong correlation between pre and post-CPM clamp pain intensity $(\rho=0.81, P>0.0001)$, moderate correlation between clamp pain and PPT $(\rho=-0.43, P>0.0001)$, as well as CPT pain intensity $(\rho=0.32, P>0.0001)$ and a weak correlation with time to onset of CPT pain $(\rho=-0.14, P<0.05)$.

The application of mechanical pressure is commonly employed to assess general pain sensitivity, and a simple spring-clamp seems a potentially useful instrument for quantitative sensory testing in a clinical setting.

Keywords: Experimental pain, Quantitative sensory testing, Low-back pain, Central sensitization, Generalized hyperaglesia, Pain sensitivity

\footnotetext{
* Correspondence: soeren.oneill@rsyd.dk

'SMI Laboratory for Human Experimental Pain Research, Center for

Sensory- motor Interaction, Department of Health Science and Technology,

University of Aalborg, Fredrik Bajers Vej 7, Bld. D3, 9220 Aalborg, Denmark

${ }^{2}$ RCF Spine Center of Southern Denmark, Lillebælt Hospital, Østre Hougvej

55, 5500 Middelfart, Denmark

Full list of author information is available at the end of the article
}

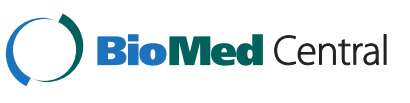

(c) 2014 O 'Neill et al.; licensee BioMed Central Ltd. This is an Open Access article distributed under the terms of the Creative Commons Attribution License (http://creativecommons.org/licenses/by/2.0), which permits unrestricted use, distribution, and reproduction in any medium, provided the original work is properly credited. The Creative Commons Public Domain Dedication waiver (http://creativecommons.org/publicdomain/zero/1.0/) applies to the data made available in this article, unless otherwise stated. 


\section{Background}

Assessing general pain sensitivity is clinically relevant, as generalised hyperalgesia appears to be an important factor in the chronification of many pain conditions, including e.g. low back pain (LBP) [1-5]. However standardized assessment of pain sensitivity and central modulation of pain sensitivity is probably used only rarely in clinical practice. Chiropractors and other healthcare providers who deal with musculoskeletal pain routinely assess mobility, muscular function, neurological integrity and similar aspects of the patient presentation in semi-standardized ways as taught in pre-graduate training and described in common textbooks. In contrast, no corresponding standardized method of quantifying pain sensitivity and modulation locally, segmentally or generally have been described for musculoskeletal problems and we suspect such examination procedures are not in common clinical use. Subjective patient reports of widespread pain, abnormal pain descriptions and disproportionate exacerbations with physical activity, may alert the clinican to potential abnormal pain sensitivity and modulation, and such suspicions should arguable be documented by quantitative sensory testing (QST).

Like clinical tests of e.g. spinal mobility and deep tendon reflexes, QST should be safe, easy and fast to perform and ideally should not rely on expensive specialist equipment. Otherwise it is unlikely they will be used in busy clinical practice. Unfortunately, the range of experimental pain stimuli which are available in the laboratory, are generally prohibitively expensive and/or difficult to use in a clinical setting. Such laboratory procedures include deep and superficial electrical stimulation, injection or topical application of noxious substances, controlled mechanical or thermal stimuli and many others. For the most part these require considerable training and experience to perform and interpret, and furthermore they often require expensive equipment such as constant current stimulators or peltier thermodes.

Different QST procedures measure different aspects of pain sensitivity, whilst others overlap to some degree [6-8]. The pressure algometer is often used to assess pain detection thresholds of deep-tissue structures [9], but quantifying the effects of temporal summation or conditioned pain modulation $(\mathrm{CPM})$, require a more complicated set-up [10]. In any case, a single pain test is most likely insufficient to get an adequate picture of pain sensitivity in a complex clinical context, where different pathways and mechanisms are involved. Rather, it is a question of choosing the right selection and combination of QST procedures [6-8]. Obviously, the more QST procedures in such a selection of tests, the more expensive, time-consuming and difficult it becomes and therefore less likely to be adopted in clinical practice.
Mechanical pressure pain sensitivity of the thumb has been advocated as a good choice of a 'neutral' site for testing general pain sensitivity - i.e. a site at which general pain sensitivity can be assessed, unaffected by any localized tissue pain such as e.g. low-back pain [3,11]. Clauw et al. [4] reported a moderate correlation $(\rho=0.38-0.52)$ between pressure pain detection and tolerance thresholds of the thumb and physical function and pain in LBP sufferers. Pain sensitivity of the thumb and thenar muscles have also been used as an indicator of general pain sensitivity in reports of hyperalgesia in relation to vulvodynia [12], the peri-menstrual period [13] and myofacial jaw pain [14]. Giesecke et al. [3] used mechanical pressure of the thumb in a study of chronic LBP and fibromyalgia to demonstrate increased pain sensitivity compared to pain-free controls.

To adequately assess pain sensitivity, the clinician would most likely need a battery of carefully selected pain tests. In this study, we examined the reliability of an in-expensive spring-clamp, which could potentially be useful as one such test in a battery of clinically applicable standardized QST in the assessment of general pain sensitivity.

\section{Methods}

\section{Subjects and materials}

It was our deliberate intention to include a heterogeneous group of participants with or without low-back pain, of different characteristics (chronic/acute, with/without leg pain, etc.). For that reason, participants were recruited from three different populations: Hospital LBP outpatients, LBP patients in primary sector chiropractic clinics, and healthy volunteers.

By virtue of the free access to chiropractic care in the primary sector and the restrictive referral criteria which apply to hospital referral, hospital patients tend to be more chronic and severely affected. This was confirmed by the current data (details published elsewhere [2]).

Patients were recruited consecutively on pre-defined, pseudo-random days in both the primary care and hospital settings. No particular inclusion criteria were applied apart from low-back pain being the primary complaint.

A total of 242 participants were recruited. 127 woman and 115 men, 119 hospital patients, 79 patients from primary care and 44 healthy volunteers. Mean age was 45.4 years (95\% CI 43.8-47.0).

Hospital patients, primary chiropractic care patients and healthy volunteers are collectively referred to as QST participants hereafter. In addition to QST participants, 20 (healthy, pain free) experimenters were recruited to perform reliability and dose-response measurements of the spring-clamp.

The current study was concerned mainly with the reliability and validity of the spring clamp as an experimental 
pain stimulus with potential clinical applicability. Parts of the data has also been analysed and published in another manuscript focused on differences in pain sensitivity between clinical subgroups [2].

The experiment was conducted in accordance with the Helsinki Declaration and IASP guidelines for human pain research [15]. Approval was granted by The Regional Committees on Health Research Ethics for Southern Denmark (ref. S-20090003).

\section{Clinical data}

Hospital patients and primary care patients were asked to indicate average, current and worst LBP intensity (with or without radicular pain) on $100 \mathrm{~mm}$ paper VAS (0-100) and healthy volunteers were asked to confirm, that they did not suffer any pain complaints, after which QST was performed. Furthermore low-back pain patients (hospital and private practice) completed the RolandMorris disability questionnaire [16] and EQ5D forms [17] for descriptive purposes.

\section{Experimental QST protocol}

QST consisted of pressure pain detection threshold (PPT) of the left infraspinatus muscle, spring-clamp pain intensity with 10 seconds sustained pressure over the left thumb nail-bed, conditioned pain modulation (CPM) with cold-pressor test (CPT) as the conditioning stimulus and (repeat) spring-clamp pain intensity as the test stimulus. Spring-clamp pain was re-tested at the first subsequent consultation for hospital patients.

QST data was collected by SON or one of two research assistants. Prior to data collection both assistants (senior clinical interns) were instructed in the correct QST procedure and all three examiners practised the procedures together until confident they were performed as uniformly as could reasonably be expected. No formal testing of variation between testers was performed.

\section{Pressure pain threshold}

Pressure was applied manually with an algometer (Somedic pressure algometer with a $1 \mathrm{~cm}^{2}$ probe, model 2, Hørby, Sweden) on the left Infraspinatus muscle, with a near-constant velocity of $50 \mathrm{kPa} / \mathrm{s}$ until the subject indicated the pressure as becoming painful by pressing an indicator button connected to the algometer.

PPT measurements were repeated 3 times with 5-10 seconds interval and the average was used for further analysis. The probe of the algometer was placed such that repeat applications overlapped partly. If no pain had been elicited by $1000 \mathrm{kPa}$, this was recorded as the PPT. If the first and second measurement was $1000 \mathrm{kPa}$, a third was not performed.

\section{Clamp - sustained mechanical pressure}

The spring-clamp (Millarco $100 \mathrm{~mm}$ with two $14 \times 13$ $\mathrm{mm}^{2}$ pressure pads, Prod.Nr. 72644, Millarco, Lystrup, Denmark - see Figure 1) was placed on the left thumbnail for 10 seconds, after which participants were asked to score the pain intensity on a $100 \mathrm{~mm}$ paper VAS, marked 'No pain' and 'Worst pain imaginable' at either end (scored as 0-100 VAS).

Care was taken to ensure that the upper pad of the clamp was placed as far proximal on the nail as possible, but not overlapping the eponychium (cuticula) and the lower pad proximal enough to prevent the clamp sliding forward. In a few instances when participants had a particularly short or curved nail, it was necessary to manually prevent the clamp from sliding forward.

\section{Conditioned pain modulation}

A 25 litre water-tub (Dometic Waeco Mobicool C40, Dubai, United Arab Emirates) was kept refrigerated at 0-2 degrees Celsius and temperature was confirmed by means of a thermometer immediately before each coldpressor test.

Participants were instructed to immerse their right, non-clenched hand to the wrist in the circulating water (Reich submersible water pump, 15 l/min, 0.5 bar, Reich, Arnhem, Netherlands) and keep it there for 1 minute or until pain became unbearable. Participants were asked to verbally indicate onset of pain and time from immersion to pain-onset was noted. Similarly, time from submersion to withdrawal was recorded when participants could not tolerate the full 1 minutes coldpressor test.

Participants were informed when 30 and 50 seconds had passed and immediately after withdrawal participants indicated cold-pressor pain intensity on a $100 \mathrm{~mm}$ VAS, marked 'No pain' and 'Worst pain imaginable' at either end (scored as 0-100 VAS).

Mechanical pressure on the left thumb nail with the spring-clamp, as described above, was repeated 5-15

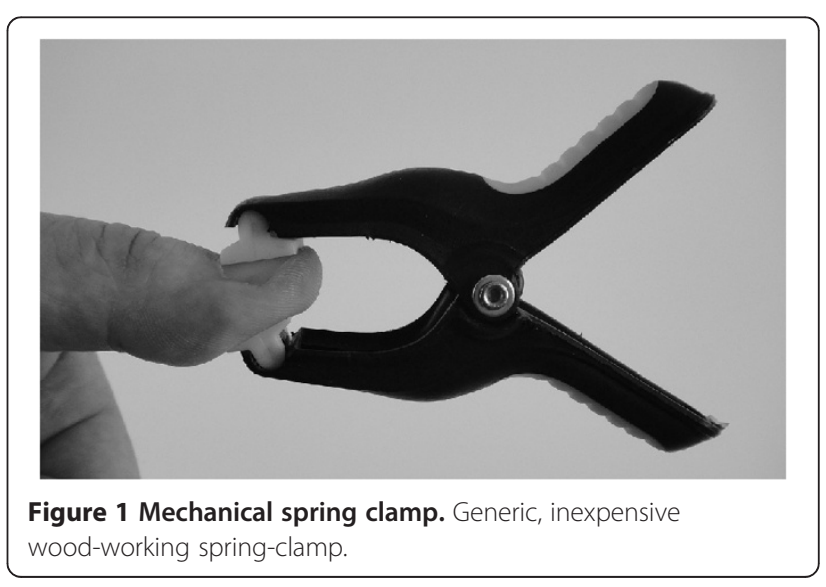


seconds after CPT, thus serving as test-stimulus in assessment of conditioned pain modulation (CPM), with $\mathrm{CPT}$ as conditioning stimulus. The same clamp was used for all QST participants, for both these tests.

\section{Test/re-test - hospital patients}

On the first subsequent visit following the initial examination, hospital patients were re-tested with a springclamp, by the attending clinician. Logistically, there was a need for several clamps for the re-test, and therefore a random clamp was selected for each patient - I.e. test and re-test were performed with two different clamps (of the same type).

No re-test was possible for primary care patients.

\section{Test/re-test and stimulus-response by experimenters}

Ten pairs of senior clinical interns were recruited as experimenters (examiner and test-subject) and instructed how to test a selection of six clamps of different types. Clamps were of different makes and models, with different sizes/forces, but all with $14 \times 13 \mathrm{~mm}^{2}$ pads - the same 6 clamps were used for test and re-test.

The clamps being used were shielded from view of the (blinded) test-subject. The examiner applied the 6 clamps in a pre-determined random order, with a pause of approximately 10 seconds between tests.

After 10 seconds of pressure application, the testsubject rated the pressure on a $100 \mathrm{~mm}$ plastic VAS. The VAS was denoted 'No sensation', 'Pain threshold' and 'Worst pain imaginable' corresponding to a score of 0 , 50 and 100, respectively. A written instruction was supplied, explaining that any non-painful sensation should be rated between 'No sensation' and 'Pain threshold', and similarly any painful sensation between 'Pain threshold' and 'Worst pain imaginable'.

Finally, the examiner measured the mechanical force generated by each of the six clamps, using a scale (Kern HDB 10 K10, Kern \& Sohn Gmbh, Balingen, Germany) in a custom made aluminium frame, with the jaws of the clamp opened to $7 \mathrm{~mm}$.

The same six clamps were re-tested, in a new random order, a few days later, by the same examiner and testsubject pairs.

\section{Variation in clamp force, including temperature and degree of opening}

To assess inter-clamp variability, the force generation of all sixty-two available, similar spring-clamps were tested once (by SON). To assess intra-clamp variability a random selection of ten clamps were tested ten times each, using the Kern scale.

Five other clamps were selected at random and submerged in 40 degree Celsius warm water. As the water cooled, the clamp force was measured at 40, 35, 30, 25 and 20 degrees Celsius.

Finally ten clamps were randomly selected and, using a set of $1.5 \mathrm{~mm}$ thick washers and the Kern scale, the clamp force was measured with the jaws of the clamps opened to $7,8.5,10,11.5$ and $13 \mathrm{~mm}$.

\section{Statistical analysis}

Data was analysed using $\mathrm{R}$ version 2.13.1 (R Foundation for Statistical Computing, Vienna, Austria) for Linux and an P-level of 0.05 was accepted as significant. Pvalues are reported numerically or by star-annotation, where * is $\mathrm{P}<0.05, *$ is $\mathrm{P}<0.01, * * *$ is $\mathrm{P}<0.001$ and $* * *$ is $\mathrm{P}<0.0001$.

Non-parametric analysis was employed: Summary statistics are reported as median value $(95 \% \mathrm{CI})$ [inter-quartile range], except where otherwise stated. Correlations were calculated as Spearmans $\rho$ and reliability of repeat measurements were analysed with Limits of Agreement.

\section{Results}

\section{Summary of clinical data}

The distribution of VAS scores for average, current and worst low-back pain is illustrated in Figure 2.

Eighteen low-back pain patients (and 44 healthy volunteers) did not report duration of low back pain. Of the remaining, 58 reported LBP for 3 months or less and 122 reported LBP for more than 3 months. 67 participants reported LBP for more than a year.

Of 198 low-back pain patients, 22 did not complete the Roland-Morris disability questionnaire, nor the Quality of Life (EQ5D). The median EQ5D score (adjusted to the Danish population norm) was 0.723 (IQR [0.59;0.82]) and the median Roland-Morris score was 10 (IQR $[4 ; 15]$ ).

\section{Quantitative sensory testing}

The results of QST are illustrated in Figure 3. Median pressure pain threshold was $447 \mathrm{kPa}$ (IQR [341;588]) (in 8 participants PPT was right-censored as $1000 \mathrm{kPa})$, median VAS score with clamp before CPT was 21 (IQR [7;41]), median clamp VAS after CPT was 13 (IQR [4;30]), median time to onset of pain with CPT was 12 seconds (IQR [8;19]), median CPT VAS score was 73 (IQR $[59 ; 85]$ ) and median time to discontinuation of CPT was 60 seconds (IQR [34;60]).

\section{Clamp pain correlation to other pain modalities}

Bi-plots of correlation between clamp pain (before CPM) and other QST are illustrated in Figure 4. A moderate correlation was found between clamp pain intensity and PPT $(\rho=-0.43, \mathrm{P}<0.0001)$ and CPT pain $(\rho=0.32, \mathrm{P}<0.0001)$. A weak correlation was found between clamp pain and time from start of CPT until CPT pain $(\rho=-0.14$, $\mathrm{P}<0.05)$ and a strong correlation was found between 

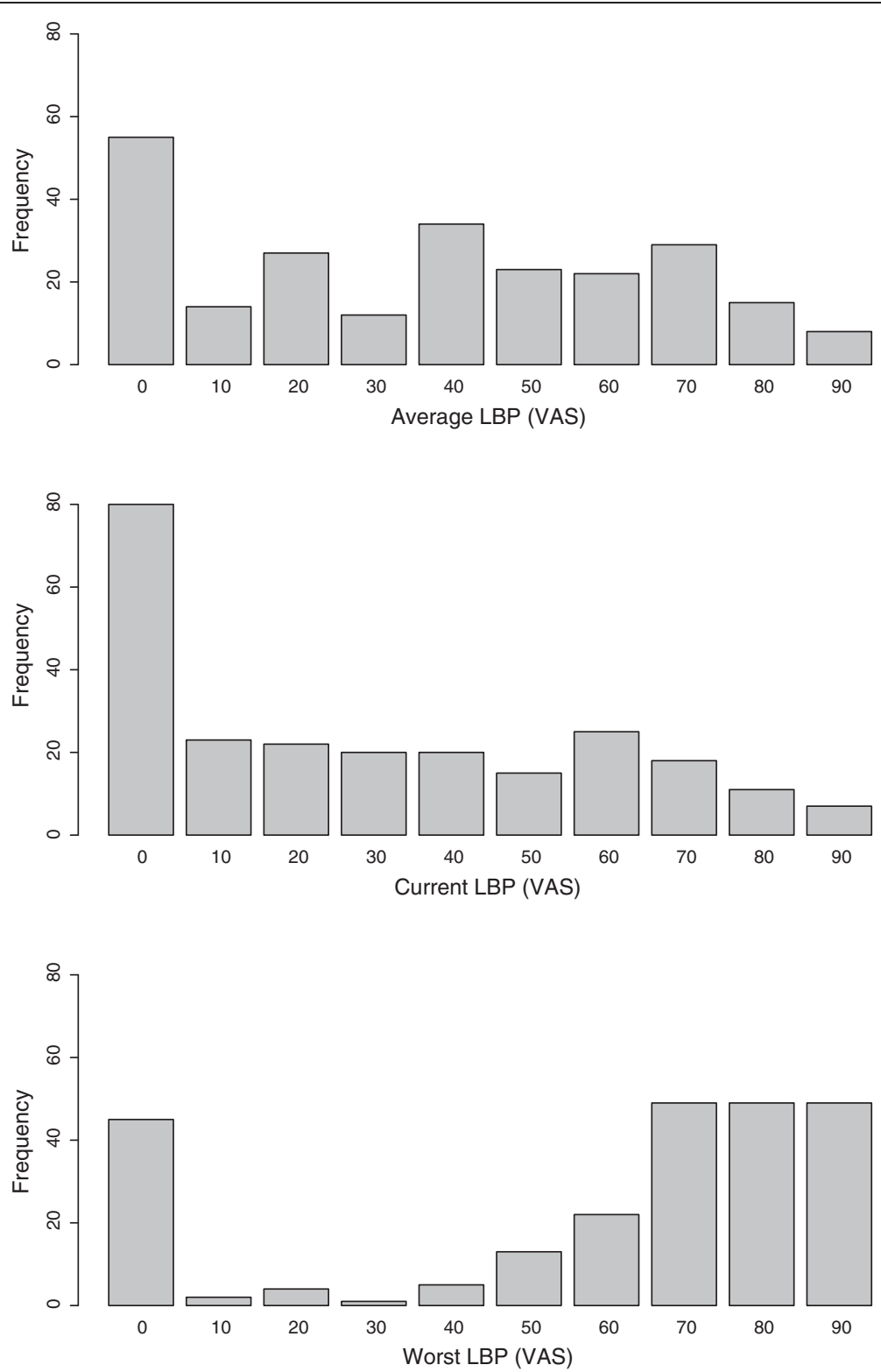

Figure 2 Low back pain intensity. Distribution of average, current and worst low-back pain intensity.

clamp pain intensity before and after CPT $(\rho=0.81$, $\mathrm{P}<0.0001)$.

\section{Conditioned pain modulation}

Spring-clamp pain dropped significantly after conditioned pain modulation (Wilcoxon signed rank test, $\mathrm{p}<0.0001$ ). The median change in absolute VAS score was only 3 (IQR $[0.0 ; 13.8]$ ), but the median relative change in VAS score was 27.2\% (IQR [0.0;62.8]).

\section{Test/re-test}

The experimenters (examiners and test-subjects) re-tested the set of 6 clamps 1-21 days (mean 6.5) after the first test. The Limits of agreement plot illustrates a mean difference in test/re-test VAS scores of 1.84 ( $S D=13.81)$. See upper-left panel of Figure 5.

Similarly, re-test clamp VAS scores were obtained for 81 hospital patients. Re-test was performed 5-154 days (mean 35) after the first test. Mean difference was 1.54 $(S D=24.45)$. See upper-right panel of Figure 5 .

\section{Stimulus/response}

A stimulus-response plot of the mechanical pressure of the 6 different clamps and the corresponding VAS scores is illustrated in the lower right-hand frame of Figure 5. A strong and highly significant correlation was found $(\rho=0.73, \mathrm{P}<0.0001)$. 


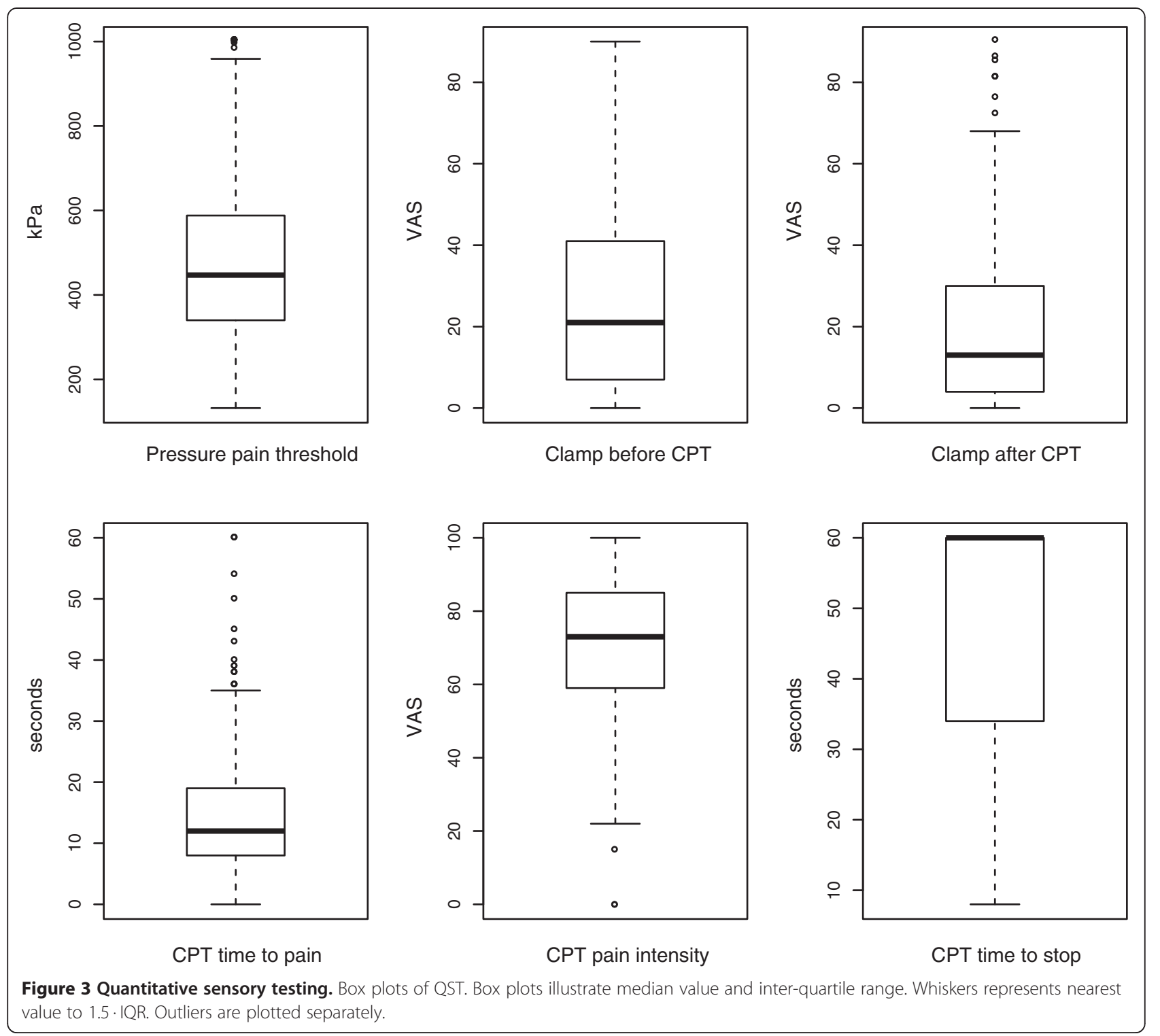

\section{Variation between and within clamps}

The force generated by the 62 available clamps of similar design, is illustrated in Figure 6. The median clamp force was $5.8 \mathrm{~kg}(4.8-6)$ [3.5;6.4].

On repeated testing of 10 random clamps 10 times, the standard deviation (normalized relative to each clamp) was $0.04 \mathrm{~kg}$.

\section{Variation with temperature and degree of clamp opening} A strong linear relationship between degree of clamp jaw opening $(7-13 \mathrm{~mm})$ and generated clamp force was found. Conversely, temperature and clamp force did not correlate. See Figure 7.

\section{Discussion}

\section{Mechanical properties}

Individual clamps reliably re-produced a consistent force on re-test with an SD of only $0.04 \mathrm{~kg}$. By contrast there was considerable variation between apparently identical clamps of the same model and the distribution was not normally distributed, as illustrated in Figure 6.

The clamp force was not affected notably by temperature variations, but was affected by the degree of clamp jaw opening (as would be expected). With the clamps used in the present study, a difference of $4 \mathrm{~mm}$ in clamp jaw opening translated into a change in clamp force of approximately $1 \mathrm{~kg}$, which suggests that variations in clamp jaw opening should be either 

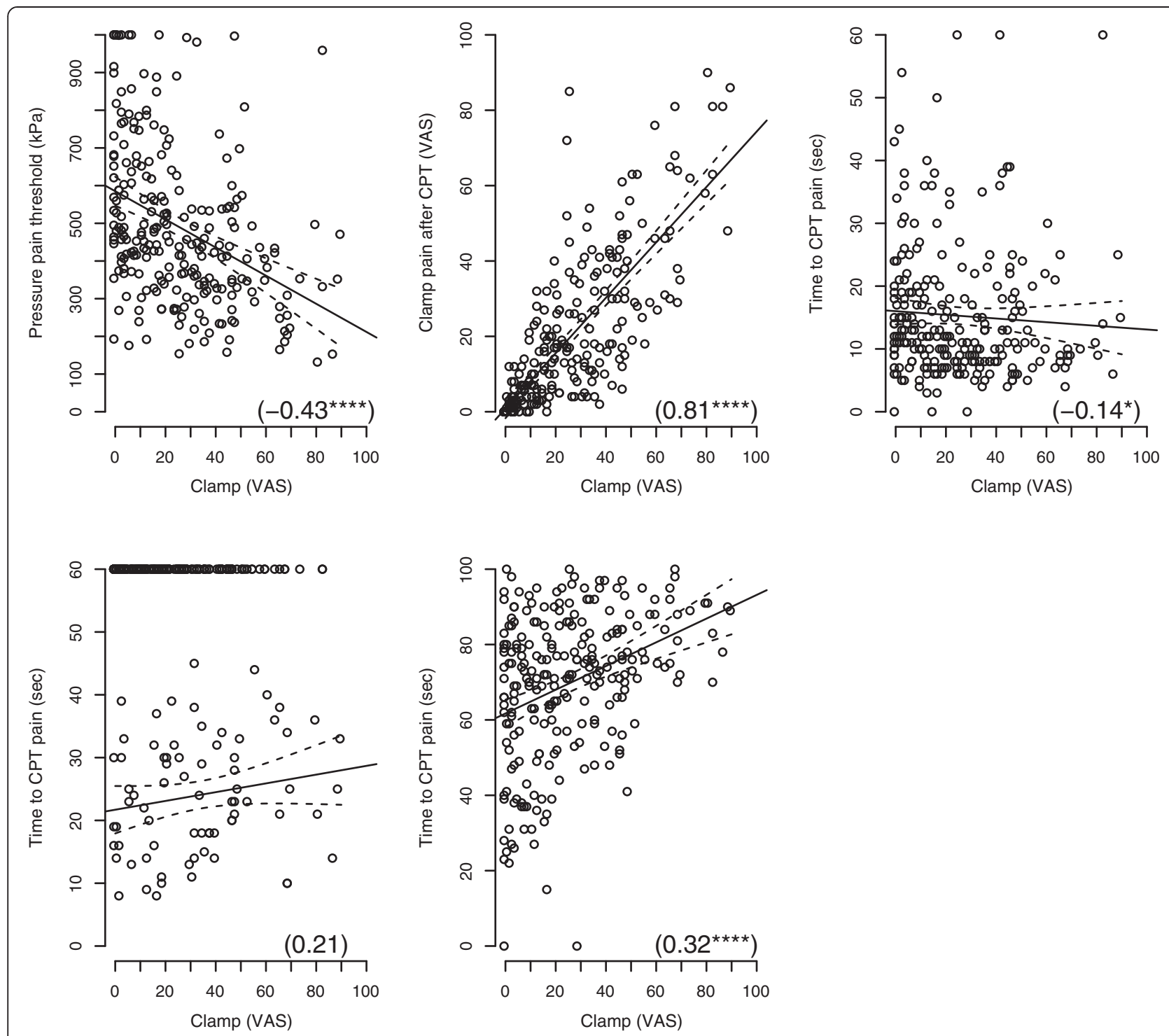

Figure 4 Spring clamp pain and other QST. Linear regression of spring-clamp VAS score and other pain stimuli (with 95\%Cl). Data was obtained from all participants $(n=242)$. Numbers in lower-right corner of plot area are Spearmans $\rho$ with statistical significance level (star-annotation). For the CPT time to discontinued, all data have been plotted, but only observations $<60$ seconds have been included in regression and correlation analysis.

controlled or reported in future studies employing a spring-clamp.

\section{Properties as a pain stimulus Stability}

Clamp pain was more stable over a period of days in healthy volunteers, compared to chronic hospital patients over a period of weeks: Arguably because of greater inherent variability in chronic pain patients and potentially also because of the greater time lapse between tests. Furthermore the use of two different clamps at test and re-test in hospital patients is likely to induce greater variation - As the current data demonstrate, there is notable variation in clamp force between individual clamps of the same make and model. This suggests that the force should be recorded if spring clamps are to be used in a clinical setting or for further research.

\section{Correlation}

The clamp pain was correlated in varying degrees to other QST: clamp pain score after CPM, PPT, CPT pain score and time from CPT start until CPT pain onset. No correlation was found to CPT tolerance. In other words, clamp pain intensity correlated to other QST to varying degrees, which is in line with the literature which suggests, that 


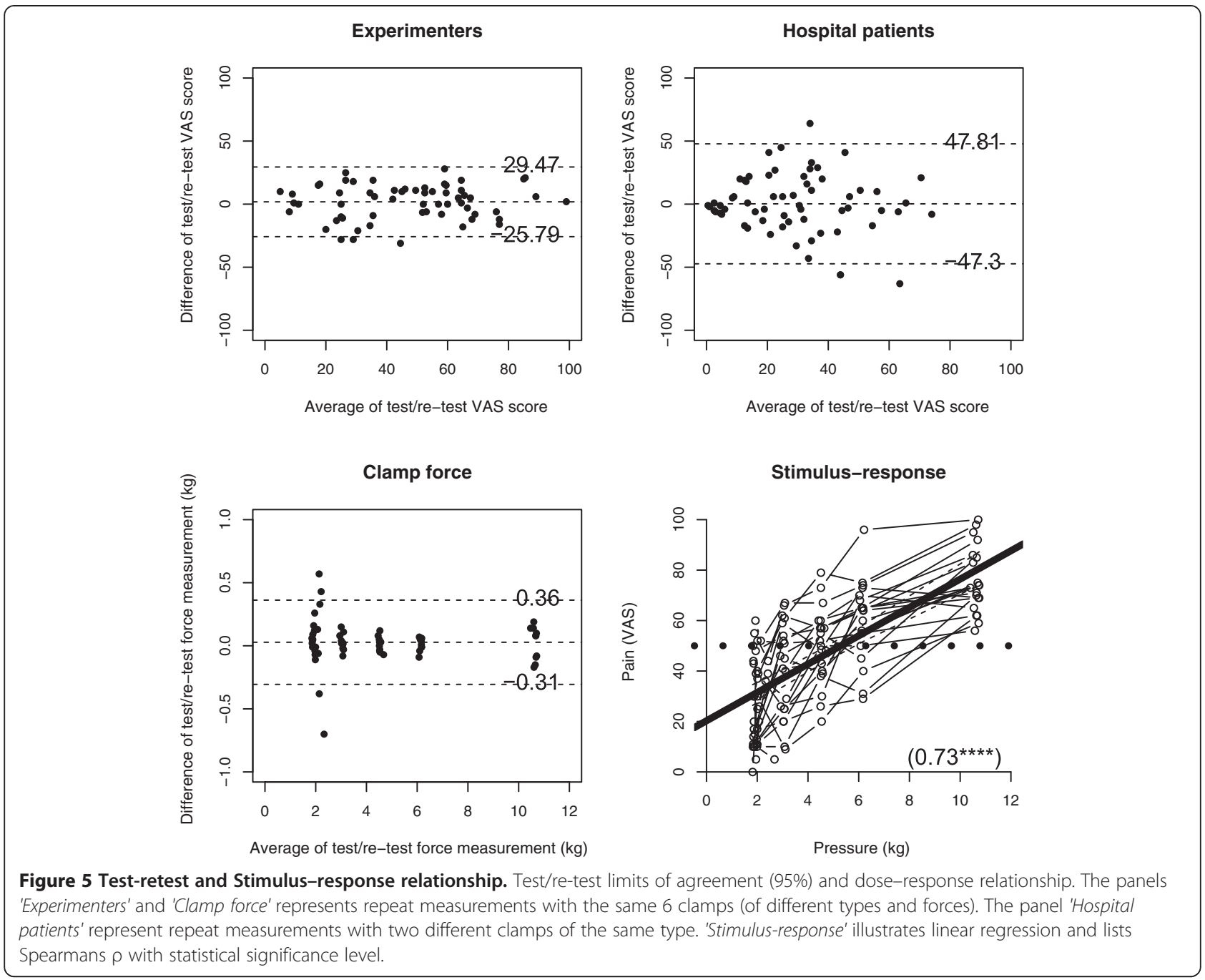

different methods of QST measure different aspects of pain sensitivity and modulation $[6,7,18,19]$.

\section{Applicability}

Unlike the algometer, the spring-clamp can only be used to apply a fixed, sustained pressure but as spring-clamps are commercially available in a large variety of sizes, it should be possible to prepare a set of clamps of gradually increasing force for clinical use. By applying 6 different clamps in what is essentially a multiple random staircase model [20], we observed a clear dose-response relationship between pain scores and clamps-forces.

If only a single clamp is used, that particular clamp should be of sufficient calibre/strength to actually induce pain in the majority of study subjects - a pressure in the range of $5-10 \mathrm{~kg}$ with an application pad area of $1-2 \mathrm{~cm}^{2}$ seems a good candidate when applied to the thumb.

A further consideration is the tissues to which the spring-clamp can be applied, which is limited by the degree of opening permitted by the spring-clamp jaws. It is feasible to apply a spring-clamp to skin-folds, fingers, toes, ear-lobes etc. and probably also small, peripheral muscle groups such as the thenar and hypo-thenar muscles - this has not been investigated in the current study however and the validity of the spring clamp as a pain stimulus may differ between test sites.

\section{Conditioned modulation of clamp pain}

The spring-clamp was sensitive to changes in pain sensitivity as a result of conditioned pain modulation. A significant change in spring-clamp pain intensity were observed, with the majority of participants reporting less clamp pain after conditioned pain modulation. This suggests that pain induced by the spring clamp is sensitive to changes in central pain modulation. Futhermore, the spring clamp has previously been used to examine differences in pain sensitivity between clinical subgroups (acute and chronic LBP patients and healthy controls) [2]. 


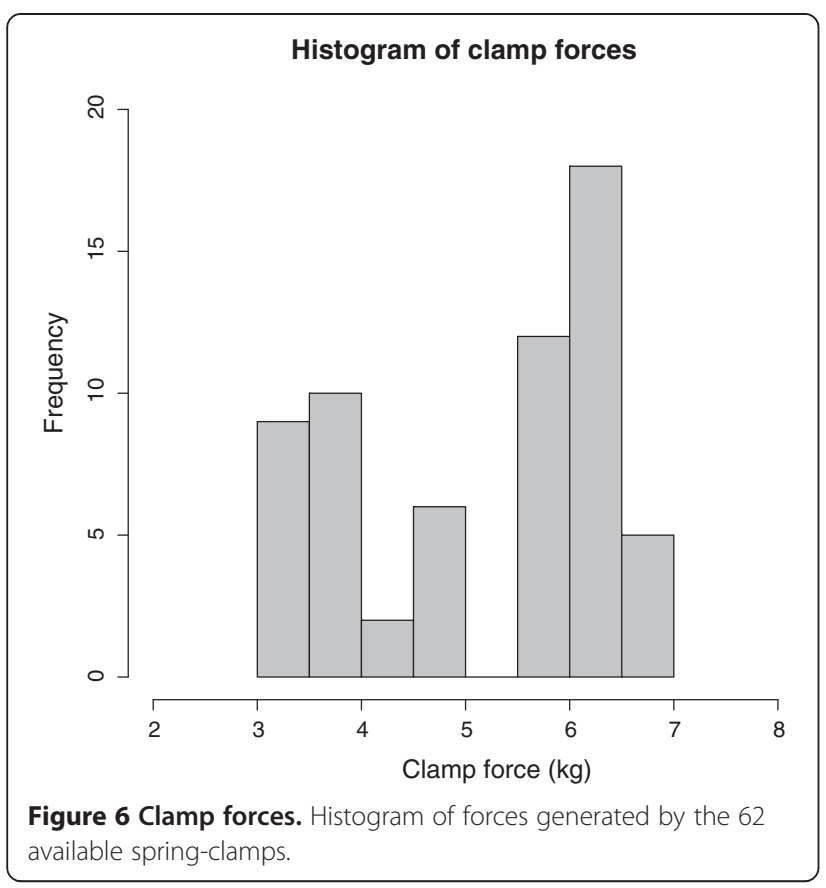

\section{Previous studies}

During data collection of the present study, Egloff et al. [21] published a similar study employing a clothes peg as the pain stimulus. The authors examined differences in pain response between two different clinical sub-groups (orthopaedic versus medical/psychosomatic pain), reported good correlation to pressure pain detection and tolerance thresholds and good test/re-test stability. The correlations between clothes-peg pain intensity and pressure pain thresholds, using a standard algometer, were reported as Spearmans $\rho$ between -0.38 and -0.62 - i.e. of similar magnitude to the current findings $(\rho=-0.43)$. All-in-all, the findings of the current study and those of Egloff et al. align.
Two other LBP studies have been published in which mechanical pain sensitivity of the thumb has been used as an indicator of general pain sensitivity [3,4], but these have used a traditional algometer to measure PPTs and a custom made apparatus with hydraulic valves and calibrated weights. To our knowledge, the study by Egloff et al. is the only other work using a simple clamp/peg technique.

\section{Perspectives for future use}

The association between chronic pain and generalized hyperalgesia is well supported in the literature and may play an important part in clinical management of painful disorders. However, establishing a reliable diagnosis of altered central pain modulation in e.g. specific LBP cases, is not yet possible [22]. If such a diagnosis is to have clinical benefits, it should be made early in the course of pain. Chiropractors in primary practice are in a position to examine such patients, early in the course of pain.

If a diagnosis is to be made based on an individuals changes in pain sensitivity over time, as opposed to population reference values, this underlines the need for quick, reliable and simple pain tests early in the clinical course of pain. The spring clamp tested in the current study could serve as one such a pain stimulus, possibly as part of a larger test battery.

\section{Conclusions}

No single QST provides a complete picture of pain sensitivity or mechanisms of central nociceptive modulation and the spring-clamp has obvious limitations, but it does offer a simple, reliable and clinically applicable pain stimulus for assessing pain patients in clinical practice. The clinical assessment and implications of such testing is still unclear.
Temperature vs. clamp force

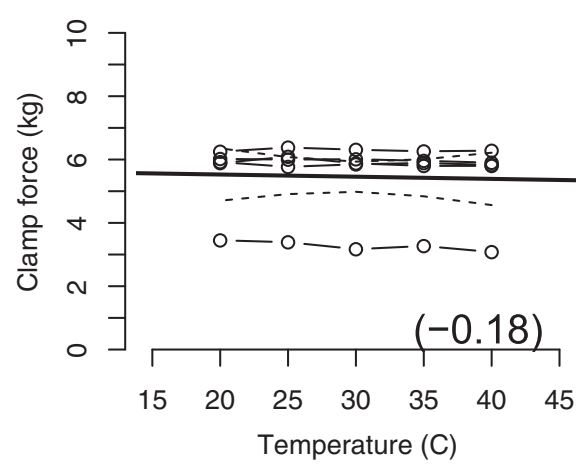

Jaw opening vs. clamp force

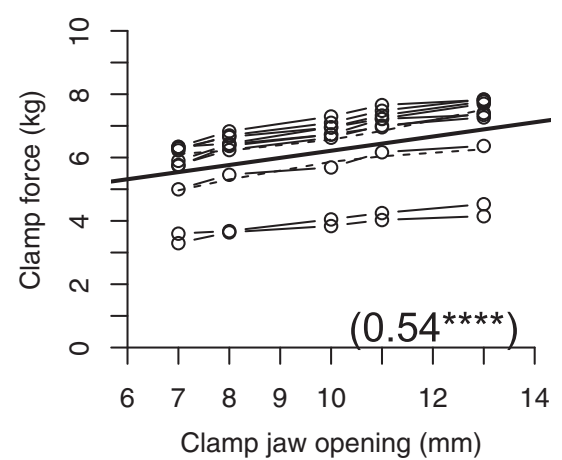

Figure 7 Effect of temperature and degree of clamp opening. Correlation of clamp force with temperature and degree of clamp jaw opening. Numbers in lower-right corner of plot area is Spearmans $\rho$ with statistical significance level (star-annotation). Five random clamps tested for sensitivity to temperature changes and 10 random clamps tested for degree of jaw opening. 


\section{Abbreviations}

CPM: Conditioned Pain Modulation; LBP: Low-back pain; PPT: Pressure Pain Detection Threshold; QST: Quantitative Sensory Testing.

\section{Competing interests}

We the authors declare no conflicts of interest or competing interests in the preparation of the present manuscript.

\section{Authors' contributions}

Author SO'N: Contributed significantly to the design of the project, manuscript authoring, data collection and analysis. Author TG-N: Contributed significantly to the design of the project and manuscript authoring. Author CM: Contributed significantly to the design of the project and manuscript authoring. Author LA-N: Contributed significantly to the design of the project and manuscript authoring. The manuscript has been approved by all 4 authors.

\section{Acknowledgements}

Contributions which do not justify authorship: none.

Technical help: none.

Financial and material support: none.

Conflicts of interest: none.

The authors state no conflicts of interest in the preparation of this manuscript and the research on which it is based.

\section{Author details}

${ }^{1}$ SMI Laboratory for Human Experimental Pain Research, Center for Sensory- motor Interaction, Department of Health Science and Technology, University of Aalborg, Fredrik Bajers Vej 7, Bld. D3, 9220 Aalborg, Denmark ${ }^{2}$ RCF Spine Center of Southern Denmark, Lillebælt Hospital, Østre Hougvej 55, 5500 Middelfart, Denmark. ${ }^{3}$ SDU Institute of Regional Health Research, University of Southern Denmark, Campusvej 55, 5230 Odense, Denmark

Received: 27 January 2014 Accepted: 27 July 2014

Published: 14 August 2014

\section{References}

1. O'Neill S, Kjær P, Graven-Nielsen T, Manniche C, Arendt-Nielsen L: Low pressure pain thresholds are associated with, but does not predispose for, low back pain. Eur Spine J 2011, 20:2120-2125.

2. O'Neill S, Manniche C, Graven-Nielsen T, Arendt-Nielsen L: Association between a composite score of pain sensitivity and clinical parameters in Low-back pain. Clin J Pain 2013. Epub Ahead of Print (doi:10.1097/ AJP.0000000000000042).

3. Giesecke T, Gracely RH, Grant MA, Nachemson A, Petzke F, Williams DA, Clauw DJ: Evidence of augmented central pain processing in idiopathic chronic low back pain. Arthritis Rheum 2004, 50:613-623.

4. Clauw DJ, Williams D, Lauerman W, Dahlman M, Aslami A, Nachemson AL, Kobrine Al, Wiesel SW: Pain sensitivity as a correlate of clinical status in individuals with chronic low back pain. Spine 1999, 24:2035-2041.

5. Puta C, Schulz B, Schoeler S, Magerl W, Gabriel B, Gabriel HHW, Miltner WHR, Weiss T: Enhanced sensitivity to punctate painful stimuli in female patients with chronic low back pain. BMC Neurol 2012, 12:98.

6. Greenspan JD, Slade GD, Bair E, Dubner R, Fillingim RB, Ohrbach R, Knott C, Mulkey F, Rothwell R, Maixner W: Pain sensitivity risk factors for chronic TMD: descriptive data and empirically identified domains from the OPPERA case control study. J Pain 2011, 12:T61-T74.

7. Hastie BA, Riley JL, Robinson ME, Glover T, Campbell CM, Staud R, Fillingim RB: Cluster analysis of multiple experimental pain modalities. Pain 2005, 116:227-237.

8. Neziri AY, Curatolo M, Nüesch E, Scaramozzino P, Andersen OK, ArendtNielsen L, Jüni P: Factor analysis of responses to thermal, electrical, and mechanical painful stimuli supports the importance of multi-modal pain assessment. Pain 2011, 152:1146-1155.

9. Finocchietti S, Takahashi K, Okada K, Watanabe Y, Graven-Nielsen T, Mizumura K: Deformation and pressure propagation in deep tissue during mechanical painful pressure stimulation. Med Biol Eng Comput 2013, 51:113-122.

10. Yarnitsky D: Conditioned pain modulation (the diffuse noxious inhibitory control-like effect): its relevance for acute and chronic pain states. Curr Opin Anaesthesiol 2010, 23:611-615.
11. Petzke F, Khine A, Williams D, Groner K, Clauw DJ, Gracely RH: Dolorimetry performed at 3 paired tender points highly predicts overall tenderness. J Rheumatol 2001, 28:2568-2569.

12. Giesecke J, Reed BD, Haefner HK, Giesecke T, Clauw DJ, Gracely RH: Quantitative sensory testing in vulvodynia patients and increased peripheral pressure pain sensitivity. Obstet Gynecol 2004, 104:126-133.

13. Isselée $H$, Laat AD, Bogaerts K, Lysens R: Long-term fluctuations of pressure pain thresholds in healthy men, normally menstruating women and oral contraceptive users. Eur J Pain 2001, 5:27-37.

14. Michelotti A, Farella M, Stellato A, Martina R, Laat AD: Tactile and pain thresholds in patients with myofascial pain of the jaw muscles: a case-control study. J Orofac Pain 2008, 22:139-145.

15. Charlton E: Ethical guidelines for pain research in humans. Committee on ethical issues of the international association for the study of pain. Pain 1995, 63:277-278.

16. Albert HB, Jensen A-M, Dahl D, Rasmussen MN: Criteria validation of the Roland Morris questionnaire. A Danish translation of the international scale for the assessment of functional level in patients with low back pain and sciatica. Ugeskr Laeger 2003, 165:1875-1880.

17. Sørensen J, Davidsen M, Gudex C, Pedersen KM, Brønnum-Hansen H: Danish EQ-5D population norms. Scand J Public Health 2009, 37:467-474.

18. Janal MN, Glusman M, Kuhl JP, Clark WC: On the absence of correlation between responses to noxious heat, cold, electrical and ischemic stimulation. Pain 1994, 58:403-411.

19. Lautenbacher S, Rollman GB, McCain GA: Multi-method assessment of experimental and clinical pain in patients with fibromyalgia. Pain 1994, 59:45-53.

20. Gracely RH, Lota L, Walter DJ, Dubner R: A multiple random staircase method of psychophysical pain assessment. Pain 1988, 32:55-63.

21. Egloff N, Klingler N, von Känel R, Cámara RJA, Curatolo M, Wegmann B, Marti E, Ferrari M-LG: Algometry with a clothes peg compared to an electronic pressure algometer: a randomized cross-sectional study in pain patients. BMC Musculoskelet Disord 2011, 12:174.

22. Curatolo M: Diagnosis of altered central pain processing. Spine Phila $\mathrm{Pa}$ 1976 2011, 36(25 Suppl):S200-S204

doi:10.1186/s12998-014-0030-y

Cite this article as: O'Neill et al:: Reliability and validity of a simple and clinically applicable pain stimulus: sustained mechanical pressure with a spring-clamp. Chiropractic \& Manual Therapies 2014 22:30.

\section{Submit your next manuscript to BioMed Central and take full advantage of:}

- Convenient online submission

- Thorough peer review

- No space constraints or color figure charges

- Immediate publication on acceptance

- Inclusion in PubMed, CAS, Scopus and Google Scholar

- Research which is freely available for redistribution 